\title{
Influenza: Quantifying Morbidity and Mortality
}

ARNOLD S. MONTO, M.D.

Ann Arbor, Michigan

From the Department of Epidemiology, University of Michigan, School of Public Health, Ann Aibor, Michigan. Requests for reprints should be addressed to Dr. Arnold S. Monto, School of Public Health, University of Michigan, Ann Arbor, Michigan 48109
Because of their dramatic impact, morbidity and mortality associated with influenza have been recognized since at least the time of Elizabeth I of England. Excess mortality has been documented since 1889, and the infamous 1918 outbreak confirmed that influenza was truly one of the last major plagues. Despite the clear recognition of large clusters of influenza activity, it is still difficult to quantify precisely the total impact of influenza morbidity and mortality, since laboratory confirmation is required for exact diagnosis. Many methods have been developed to provide estimates of the mortality associated with influenza. These methods are usually predicated on establishing expected baseline rates of mortality. Deaths in excess of these rates are then calculated-and attributed to the circulating influenza virus. In this way, groups at high risk of mortality have been defined as the eiderly and those with chronic conditions. Special-risk groups, such as those in institutions, have also been identified. The quantification of morbidity has required different approaches, and here community and family studies have made major contributions. In contrast to mortality, morbidity is most pronounced in children and young adults, and the diseases, although self-limited, are often quite severe. Although the size of the outbreaks varies, influenza infection can be documented annually. Thus, each year must be considered an influenza year.

Like the Roman god Janus with his two faces, influenza has two appearances. To the average lay person, who may not have experienced influenza for several years, the disease does not seem a major concern or problem. To those physicians who are involved with the care of the elderly or of those in institutions, on the other hand, the lethal consequences of influenza are well known. During an outbreak, many physicians come to appreciate the major disruption - and the potentially severe morbidityassociated with influenza even in the healthy. This is especially true of those who work in student health services. Given these two views of Influenza, our task is to examine the existing data and to draw conclusions so that we can better understand the methods recommended for the prevention and treatment of influenza.

\section{INFLUENZA FROM A HISTORICAL PERSPECTIVE}

The two views of influenza go back to the first clear description. During the time of Elizabeth I of England, an outbreak of what we now believe was influenza struck the Scottish court in Edinburgh [1]. The English ambassador sent a report back, making light of the suffering of certain persons, but at the same time giving clear evidence that this was a wide- 


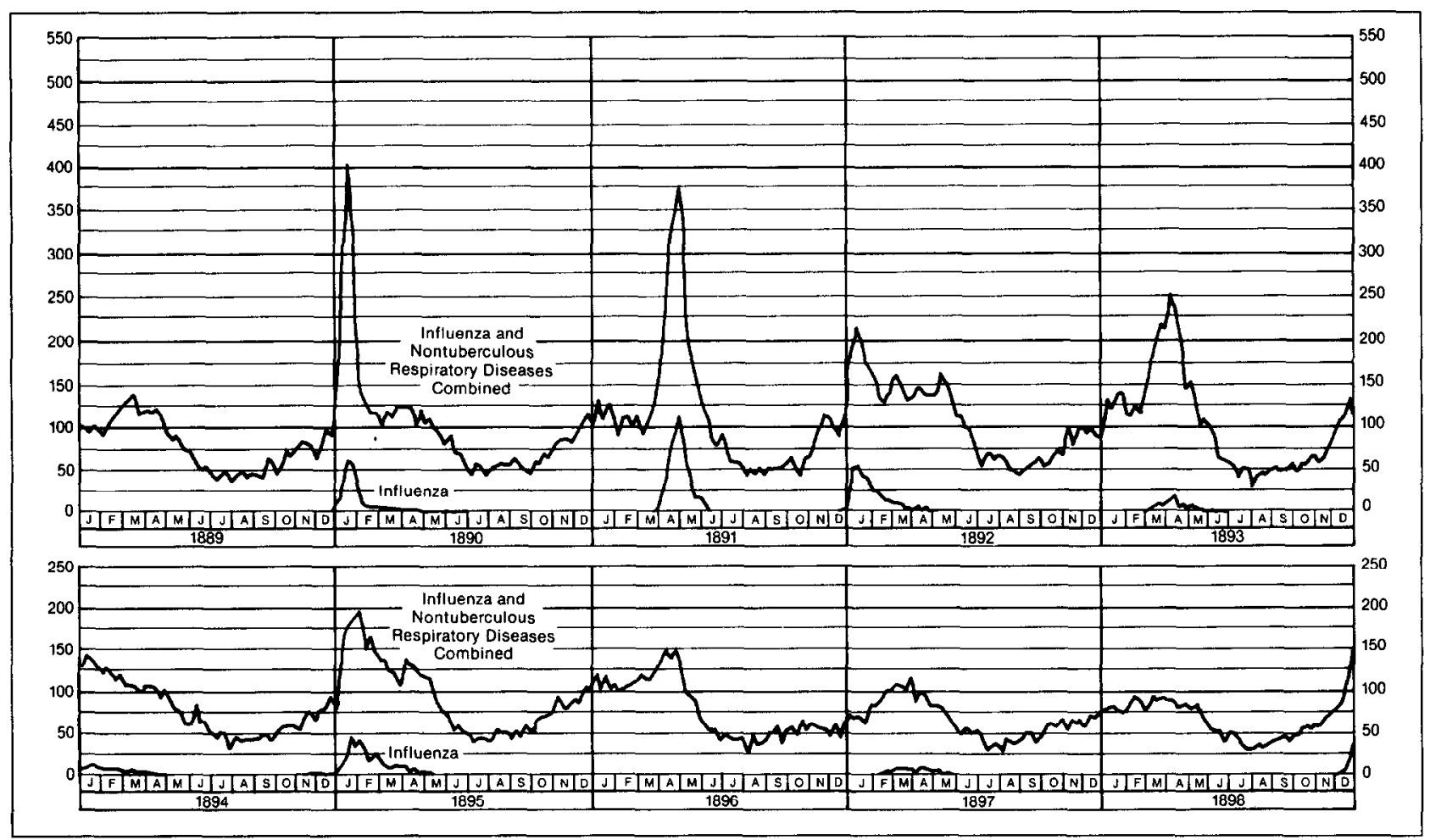

Figure 1. Weekly death rate per million of population from influenza and nontuberculous respiratory diseases in New York City, 1889 to 1898 . Data are from the Prudential Life Insurance Company.

spread disease with a high occurrence rate and considerable severity. It is precisely because of this overall impact that influenza can be tracked through history, especially when it occurred in pandemic form.

The first pandemic for which we have good public health records occurred in 1889 , and the most complete data come from certain areas of the United States as well as from the Registrar General of England and Wales. Figure 1 shows the weekly death rate in New York City during 1889-1898, as reflected in data gathered by the Prudential Insurance Company [2]. The data are presented for influenza alone and for influenza combined with other nontuberculous respiratory diseases. The curves are interesting in two respects. They clearly show both the effect of the 1889-1890 pandemic on mortality and the appearance of a second epidemic wave in 1891. They also show that death specifically attributed to influenza constituted only a small portion of the deaths from respiratory disease during the epidemic-most of which was undoubtedly influenza related. This latter finding is still apparent in today's statistics.

Epidemics of influenza were recognizable in various winters through the start of World War I, with some historians recognizing a possible pandemic in 1900. There has never been any question concerning the great pandemic of 1918, except perhaps about the likelihood of a recur- rence. The mortality in 1918 was enormous, and the estimate of deaths is approximately 20 million worldwide; 548,000 deaths occurred in the United States alone, as estimated by the United States Bureau of the Census [2]. The outbreak came at the end of the war in Europe, but deaths were not limited to the military; they occurred throughout the civilian population as well. Because of the accompanying high morbidity, essential services were heavily affected. The situation affected almost all who lived through it because of the high mortality; most families experienced either a death or a life-threatening illness.

The 1918 pandemic was a result of the appearance of a new type A influenza variant, which seroepidemiologic studies have identified as related to swine influenza. The reason behind the extreme mortality associated with these swine-like viruses has never been satisfactorily determined, but the age-specific pattern of mortality is clear. Age-specific mortality curves can be constructed for a number of influenza pandemics. All pandemics, including the 1889 outbreak previously described and those after 1918, including 1957 and 1968, showed a U-shaped pattern: modestly increased case fatality in the very young and then increasing case fatality with increasing age [3]. The 1918 outbreak was totally dissimilar, with high fatality seen in young adults. The W-shaped curve of 1918 docu- 


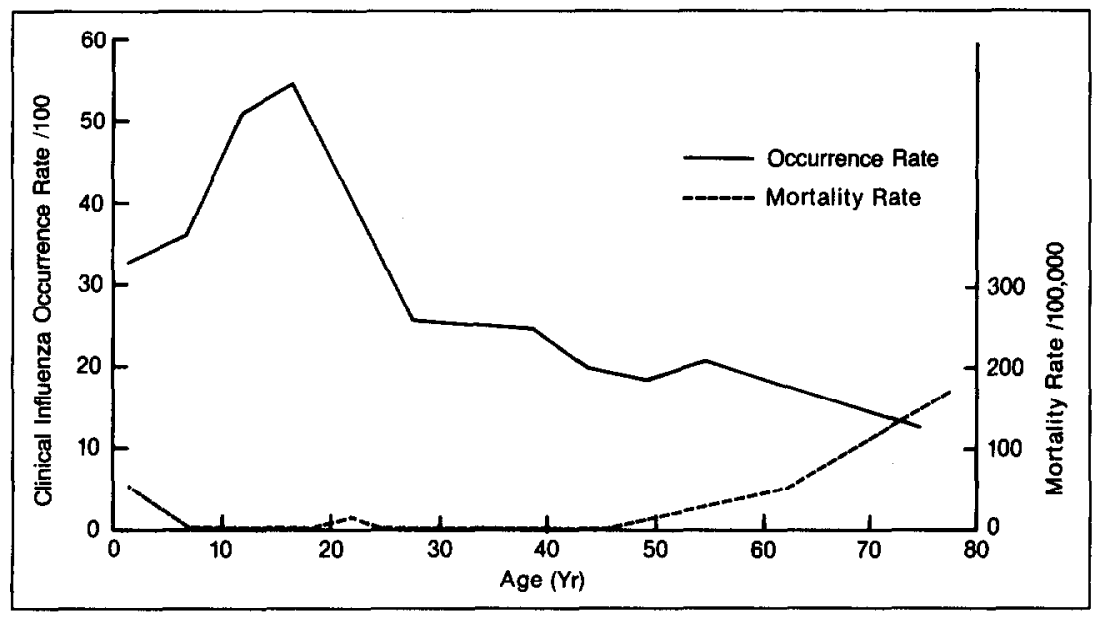

Figure 2. Clinical influenza occurrence rate (Kansas City, 1957) and annual mortality rate for pneumonia and influenza (United States, 1957).

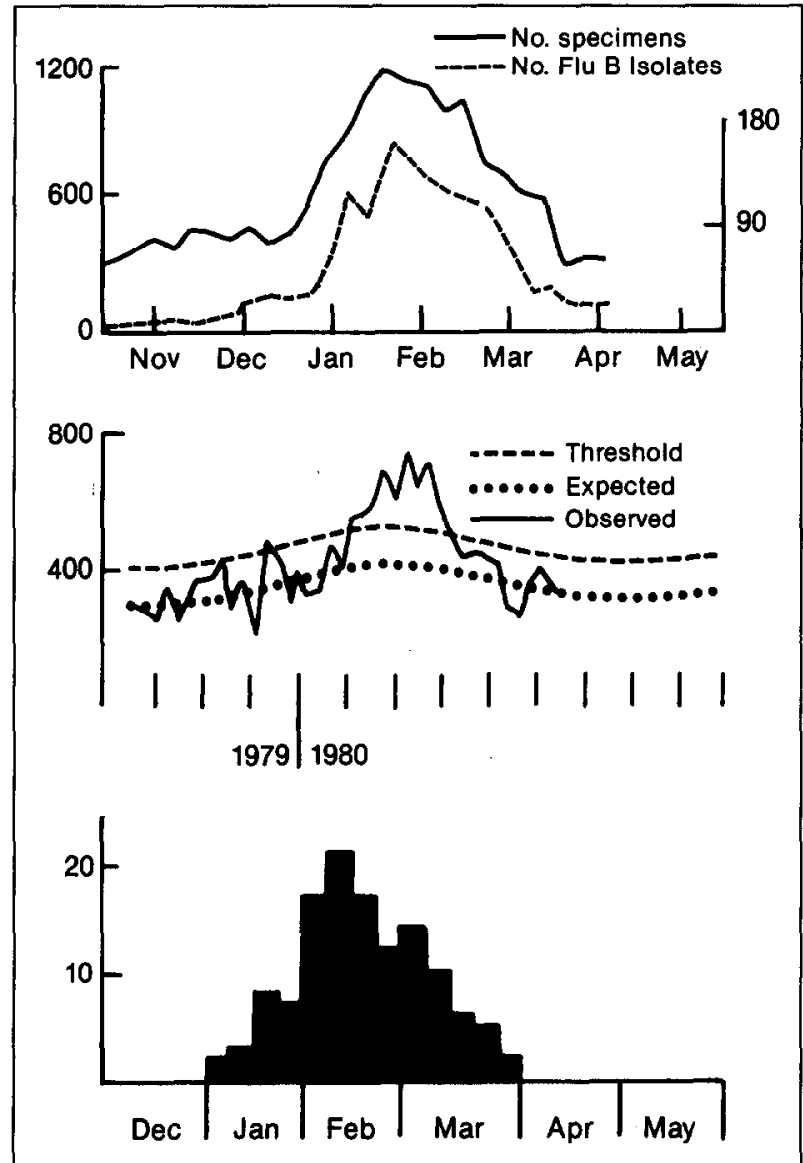

Figure 3. Influenza surveillance in the United States, 19791980. Top, laboratory surveillance for influenza infections; middle, deaths attrlbuted to pneumonia and Influenza in 117 cities; bottom, reports of influenza activity telephoned to the Centers for Disease Control.

ments the devastating impact of this pandemic, in which mortality was not restricted to extremes of age.

Following 1918, pandemics of type A viruses continued to occur-with high occurrence rates, but with mortality restricted to what are now recognized as traditional risk groups at the extremes of age. Quantification of influenza morbidity was becoming more sophisticated, and during the 1957 outbreak, clinical influenza was defined on the basis of a series of symptoms among residents of Kansas City [4]. Figure 2 shows the age-specific clinical occurrence rates; for comparison, the case fatality data in the same outbreak are also shown. We now know that clinical influenza-influenza defined by a set of symptoms-can be recognized reasonably well during an outbreak in all except the young, so the curve in Figure 2 underestimates the occurrence of disease in very young children. However, the basic pattern still holds: morbidity occurs mainly in young individuals, including young adults, whereas mortality occurs in the elderly. Thus, any policy directed toward controlling mortality only in high-risk populations will not affect the high frequency of uncomplicated disease in the balance of the population.

The 1957 and 1968 pandemics also allowed the first clear documentation of the spread of the new virus. In 1957, for instance, the disease started in the Orient, and the early spread involved countries of the Pacific basin, the Middle East, and South America [5]. Outbreaks in the United States generally did not occur until the autumn, when schools opened; an exception was a Boy Scout Jamboree, held in lowa, from which early seeding took place. Similar diagrams can be constructed for the early spread of type A (H1N1) influenza during 1977-1978.

\section{MACROEPIDEMIOLOGY OF INFLUENZA: MORTALITY}

By examining the history of influenza, we have already learned a great deal about the mortality associated with type A infections. As in all estimates of cause-specific mortality, large groups must be followed to determine the numbers involved. Nationally, a rapid monitoring of mortality is maintained by the Centers for Disease Control through weekly reporting of deaths attributed to pneumonia and influenza from approximately 120 cities throughout the country [6]. An expected number of deaths is plot- 
ted for the country as a whole and for nine regions as well, and an epidemic threshold is calculated for each. When deaths exceed this threshold, especially for a sustained time period, an epidemic is deemed to be under way.

It is important to realize that these data are based on clinical evaluation of causes of death, and not on virologic results. For example, during the years of 1973-1978, two major type A outbreaks took place: 1975-1976 and 19771978; both were reflected in excess mortality nationally. Since outbreaks are rarely uniform throughout the country, the excess mortality was observed in some regions but not in others [7]. Not seen in any of these curves is evidence for the major type $B$ activity in 1976-1977. That is because excess mortality is not a regular occurrence with type B outbreaks, as it is with type A activity. This latter relationship is so pronounced that excess mortality can be taken as an indirect indicator of type $A$ (now $A$ [H3N2]) transmission.

However, excess mortality is not always absent in type $B$ outbreaks. Once or twice every decade, type B epidemics do produce detectable excess mortality. This occurred during 1979-1980, and in Figure 3 the association of isolation of type $B$ viruses with excess mortality can be noted [8]. Also, by comparing the bottom panel, reports of outbreaks, with the panel on excess mortality, the lag in occurrence of deaths can be observed. This delay of about two weeks between onset of illnesses and death has been recognized in general for influenza for many years.

The occurrence of influenza outbreaks over time shows how excess mortality can be used as an indirect indicator of type A activity especially. These data do not tell us who is dying of influenza, although the historical data give us a strong suggestion. Contemporary studies have defined these high-risk groups: groups not necessarily at high risk of infection with influenza, but at high risk for death should they contract the disease.

All of these estimates have required the establishment of a baseline level, i.e., the frequency of the event that would have existed without the occurrence of influenza in the specific time period. A baseline is required because of the lack of specific virologic diagnosis in most of the cases. Use of such methods probably underestimates the impact of epidemic influenza, but is more precise than attributing all respiratory events in an influenza period to influenza.

Using this technique, Barker and Mullooly [9] examined severe life-threatening disease in subscribers to the Kaiser Foundation Health Plan. Data were summarized calculating the rates of hospitalization for pneumonia and inftuenza per 100,000 in two type A periods, and subtracting from each the number of deaths in a baseline reference period without influenza. As would be expected, all age and risk groups had excess hospitalizations during these periods, but the magnitude of the increase differed. The definition of high-risk conditions used was taken from the Public Health Service vaccine recommendations. Adults without high-risk conditions up to age 64 had a lower rate of excess hospitalization, whereas all persons with highrisk conditions had a much higher rate. Even those over 65 without such high-risk conditions were at varyingly but definitely increased risk of hospitalization.

These results explain the case-fatality curves that have existed since such data were available - that is, with mortality rising from age 45 because of increased numbers of persons with high-risk conditions in the population, and then increasing sharply at age 65 . They also support the basic thrust of the immunization policy in the United States, since it is directed mainly toward control of mortality.

\section{MICROEPIDEMIOLOGY OF INFLUENZA: MORBIDITY}

For many years, it has been said of influenza that many sicken but few die. We have seen that death rates can be substantial, so morbidity at these times must be very large indeed. Although estimating mortality rates has required use of relatively large populations, morbidity estimates can be made with smaller populations, in which clinical observations are supported by laboratory studies. Investigations in Houston have documented the need for careful virologic confirmation in identifying causes of morbidity [10]. The numbers of patients with respiratory diseases presenting to surveillance sites and of influenza virus isolates during the winter of 1980-1981 were compared. Disease rates peaked at the time two $A$ subtypes of influenza (A H3N2 and A H1N1) circulated. The numbers suggest that type $A$ influenza was responsible for even more than the excess above the baseline level of illnesses, but this is difficult to confirm from these data alone. The disappearance of other agents in the time period suggests that this is indeed the case.

In our studies in the community of Tecumseh, Michigan, we have combined virus isolation with identification of infection serologically, in an effort to define virus activity comprehensively. Iliness data are collected prospectively, generally by telephone call, from a sample of community residents [11]. This method allows rates of disease and infection to be calculated. Figure 4 shows viral isolation frequencies for five years of the study in specimens collected from patients with respiratory disease. The left panel shows the percentage of specific viruses in specimens showing positive results for viruses from the community surveillance. In this surveillance, illnesses of any severity were sampled. The right panel shows the same distribution in specimens collected by community physicians from their patients. The difference in frequency of influenza isolations is striking, and it is a result of the greater severity of influenza-related diseases, which lead to physician consultations. Both panels are for illnesses occurring anytime in the year, not simply for those specimens collected during influenza outbreaks. They also are for all age groups. Even in the left panel (surveillance 


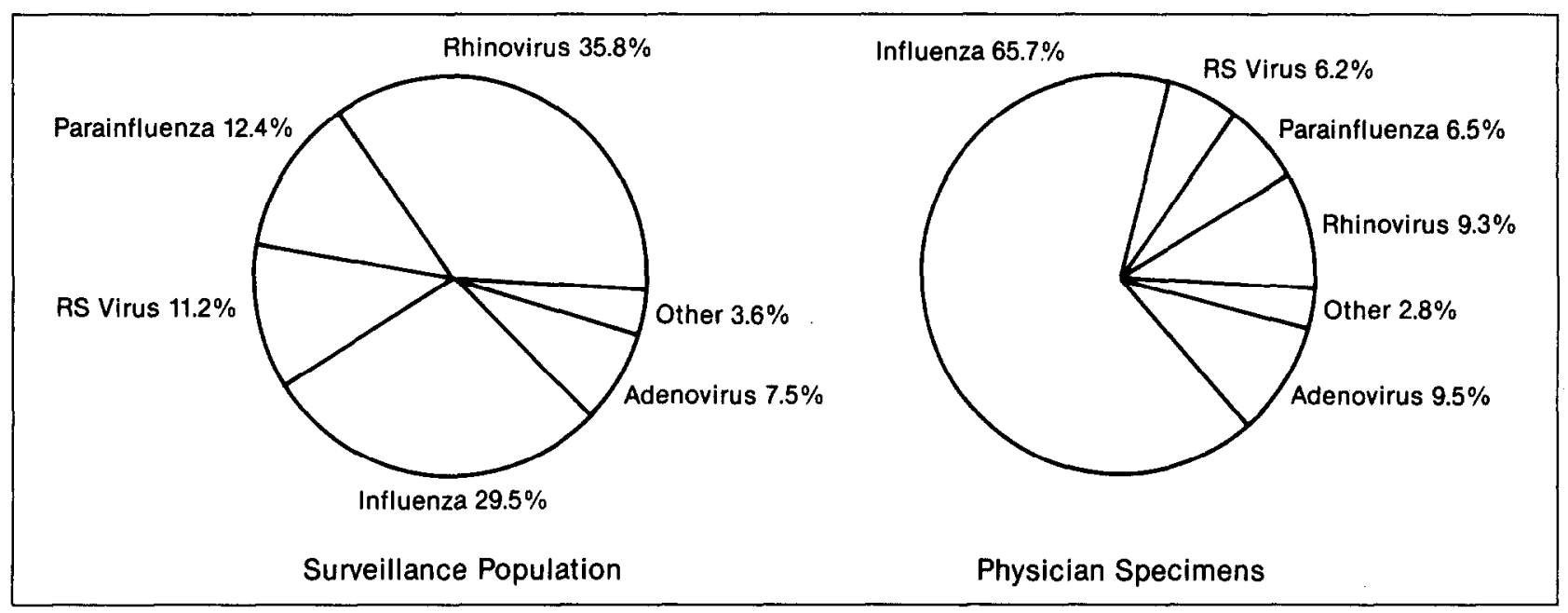

Figure 4. Percentage distribution of virus isolates, Tecumseh, Michigan, 1976 to 1981.

TABLE I Characteristics of Diseases Associated with Influenza Virus Isolates, Tecumseh, Michigan, 1976 to 1981

\begin{tabular}{|c|c|c|c|c|c|}
\hline \multirow[b]{2}{*}{$\begin{array}{l}\text { Vlrus Type and } \\
\text { Age Group (years) }\end{array}$} & \multirow[b]{2}{*}{$\begin{array}{c}\text { Number of } \\
\text { Patlents }\end{array}$} & \multicolumn{3}{|c|}{ Percent of Isolates with Indicated Disease Characteristics } & \multirow[b]{2}{*}{$\begin{array}{l}\text { Median Duration } \\
\text { (days) }\end{array}$} \\
\hline & & $\begin{array}{l}\text { Lower Resplratory } \\
\text { Disease }\end{array}$ & Fever & $\begin{array}{l}\text { Physician } \\
\text { Consultation }\end{array}$ & \\
\hline \multicolumn{6}{|l|}{ Type A (H3N2) } \\
\hline $0-19$ & 25 & 12.0 & 84.0 & 54.0 & 12 \\
\hline $\begin{array}{l}20+ \\
\text { Type A (H1N1) }\end{array}$ & \multicolumn{4}{|c|}{ Type A (H1N1) } & 15 \\
\hline $0-19$ & 47 & 12.8 & 63.8 & 25.5 & 7 \\
\hline $\begin{array}{r}20+ \\
\text { Type B }\end{array}$ & \multicolumn{4}{|c|}{ Type B } & 10 \\
\hline $0-19$ & 34 & 14.7 & 70.6 & 44.1 & 10 \\
\hline $20+$ & 17 & 52.9 & 47.1 & 41.2 & 13 \\
\hline
\end{tabular}

group), influenza isolates for all ages accounted for close to 30 percent of specimens yielding positive results. When analysis is restricted to isolates from persons of school age (ages five to 19 years) in the group under surveillance, it is found that this proportion increases to nearly 50 percent.

Another way to assess the impact of influenza is to examine the characteristics of the disease associated with virus isolation. It should be remembered that much of the clinical disease occurs in children and young adults, which can be confirmed by examining infection rates for each of the types or subtypes in question [12]. The serologic rates of infection for type A influenza (H3N2) show a high, flat pattern through childhood, remaining high but decreasing somewhat in adulthood. This calls to mind the clinically determined curve of age-specific disease from Kansas City in 1957 (Figure 2). For type B influenza, rates in the five- to 14-year-old age group were similar to those for type $A$ influenza (H3N2), but in adults the rates of type $B$ infection were much lower. For type A (H1N1), the pattern was generally similar to that of type $B$. The cut off in the mid-20s age group, at least for the clinical disease, was even more striking than with type $B$.

Referring again to disease, we see in Table I three indicators of severity determined for each influenza virus type or subtype, plus the median duration of the illnesses. Because of difference in disease characteristics by age, the results are divided into those for children and those for adults. The proportion of patients with lower respiratory symptoms (productive cough, pain on respiration, wheezing) was the same for the three virus types in children but was highest for type A (H3N2) in adults. The pattern for fever (generally more common in children than in adults) was not as consistent, but physician consultation was again most common for type $A$ (H3N2). This and the duration of disease do give an indication of the morbidity caused by influenza. The duration was longest for type $A$ (H3N2), next for type B, and shortest for type A (H1N1). 
Even here, the duration of disease was relatively longseven days in children and 10 days in the few young adults infected.

\section{COMMENTS}

Overall, there is enormous morbidity from influenza. The disease is relatively severe and leads to physician consultations. The physician must then decide how to handle a large number of cases in the course of an outbreak.

Outbreaks of influenza are easy to recognize in most cases, although in certain years transmission may be less dramatic than in others [13]. Because of the higher morbidity in the young, the existence of an outbreak is easier to recognize when young people are being seen. In any event, whether or not the outbreak is dramatic, it is important to realize that influenza occurs every winter, and that predictions of outbreaks have been notoriously poor. The national recommendations for vaccine usage acknowledge this fact by calling for annual vaccination of specific groups. The major intent of these recommendations is to control mortality by vaccinating those at high risk of death should they become infected. Higher morbidity in other populations is in part recognized by calling for vaccination of non-high-risk persons who are in close contact with high-risk persons and who could transmit the infection. Also recognized is the fact that clusters of susceptible persons increase the likelihood of transmission [14]. There is at present no recommendation for controlling the bulk of morbidity by vaccination with currently available vaccines. This means that high morbidity can still be expected to occur in outbreaks, and we must be prepared to care for those patients with appropriate therapy, which can include amantadine.

\section{REFERENCES}

1. Francis $\mathrm{T} \mathrm{Jr}$ : The new acquaintance. Ann Intern Med 1953; 39: 203-221.

2. Jordan EA: Epidemic influenza: a survey. Chicago: American Medical Association, 1927; 214.

3. Serfling RE, Sherman IL, Housworth WJ: Excess pneumoniainfluenza mortality by age and sex in three major influenza $A 2$ epidemics, United States, 1957-58, 1960, and 1963. Am J Epidemiol 1967; 86: 433-441.

4. Chin TDY, Foley JF, Doto IL, Gravelle CR, Weston J: Morbidity and mortality characteristics of Asian strain influenza. Public Health Rep 1960; 75: 149-158.

5. Francis TF Jr: Influenza. In: Rivers TM, Horsfall FL Jr, eds. Viral and rickettsial infections of man, 3rd ed. Philadelphia: J.B. Lippincott Co., 1959.

6. Housworth $\mathrm{J}$, Langmuir AD: Excess mortality from epidemic influenza, 1957-1966. Am J Epidemiol 1974; 100: 40-48.

7. Centers for Disease Control: Current trends: influenza, United States. MMWR 1978; 27: 473.

8. Nolan TF Jr, Goodman RA, Hinman AR, et al: Morbidity and mortality associated with influenza $B$ in the United States,
1978-80. A report from the Centers for Disease Control. J Infect Dis 1980; 142: 360-362.

9. Barker WH, Mullooly JP: Impact of epidemic type A influenza in a defined adult population. Am J Epidemiol 1980; 112: 798811.

10. Glezen WP: Serious morbidity and mortality associated with influenza epidemics. Epidemiol Rev 1982; 2: 26-44.

11. Monto AS, Napier JA, Metzner HL: The Tecumseh study of respiratory illness. I. Plan of study and observations in syndromes of acute respiratory disease. Am J Epidemiol 1971; 94: 269-279.

12. Monto AS, Koopman JS, Longini IM: Tecumseh study of illness. XIII. Influenza infection and disease 1976-1981. Am J Epidemiol 1985; 121: 811-822.

13. Monto AS, Kioumehr F: The Tecumseh study of respiratory illness. IX. Occurrence of influenza in the community 1966 1971. Am J Epidemiol 1975; 102: 553-563.

14. Patriarca PA, Weber JA, Parker RA, et al: Risk factors for outbreaks of influenza in nursing homes. Am J Epidemiol 1986; 124: $114-119$. 